\title{
molecules
}

ISSN 1420-3049

www.mdpi.org/molecules

Article

\section{The Antioxidant Activity and Their Major Antioxidant Compounds from Acanthopanax senticosus and $A$. koreanum}

\author{
Young-Hyun Kim ${ }^{1, \dagger}$, Myoung Lae Cho ${ }^{\text {1, }}$, Dan-Bi Kim ${ }^{1}$, Gi-Hae Shin ${ }^{1}$, Jin-Ha Lee ${ }^{1}$, \\ Jong Seok Lee ${ }^{2}$, Sun-Ok Park ${ }^{3}$, Sang-Jong Lee ${ }^{3}$, Hyun Mu Shin ${ }^{4}$ and Ok-Hwan Lee 1,*
}

1 Department of Food Science and Biotechnology, Kangwon National University, Chuncheon 200-701, Korea; E-Mails: vvkyh@naver.com (Y.-H.K.); meanglae@naver.com (M.L.C.); danbekim22@nate.com (D.-B.K.); cordelia162@hanmail.net (G.-H.S.); tre98@hanmail.net (J.-H.L.)

2 National Institute of Biological Resources, Incheon 404-708, Korea;

E-Mail: jongseoklee78@gmail.com

3 STR Biotech Co., LTD, Gangwon 200-160, Korea; E-Mails: lovastar@strbiotech.co.kr (S.-O.P.); 1sj@strbiotech.co.kr (S.-J.L.)

4 Department of Pathology, University of Massachusetts Medical School, Worcester, MA 01605, USA; E-Mail: hyunmu.shin@umassmed.edu

$\dagger$ These authors distributed equally to this work.

* Author to whom correspondence should be addressed; E-Mail: loh99@kangwon.ac.kr; Tel.: +82-33-250-6454; Fax: +82-33-259-5565.

Academic Editor: Maurizio Battino

Received: 19 May 2015 / Accepted: 15 July 2015 / Published: 22 July 2015

\begin{abstract}
The antioxidant activity and chlorogenic acid and caffeic acid contents were investigated from different parts of Acanthopanax senticosus and A. koreanum. Antioxidant activity was assessed by various in vitro assays such as DPPH, ABTS, FRAP, reducing power assays and ORAC, and the chlorogenic acid and caffeic acid were validated by HPLC chromatography. Among the various extracts, the fruit extracts of $A$. senticosus and A. koreanum exhibited strongest antioxidant activities including ABTS, FRAP, reducing power and ORAC, however, strongest DPPH radical scavenging activity was observed from the leaf extract of $A$. senticosus. In addition, the antioxidant activities of various extracts were correlated with total phenolic and proanthocyanidin contents. The major phenolic contents from various parts of these plants observed that leaf extract of $A$. senticosus expressed higher levels of chlorogenic acid (14.86 mg/dry weigh g) and caffeic acid (3.09 mg/dry weigh g) than other parts. Therefore, these results suggest that the leaf of $A$. senticosus may
\end{abstract}


be an excellent natural source for functional foods and pharmaceutical agents, and the validated method was useful for the quality control of $A$. senticosus.

Keywords: Acanthopanax senticosus; method validation; antioxidant activity; chlorogenic acid; caffeic acid

\section{Introduction}

The Acanthopanax species, belonging to the Araliaceae family, are widely distributed in the Far Eastern region of Russia and North-East of Asia countries such as Korea, Japan, China, and known as powerful tonic and medicinal herbs. These herbal plants have been attracting attention in the last few years because of their strong bioactivities, harmlessness, and low side effects [1-5]. Acanthopanax senticosus is called Eleutherococcus senticosus, Siberian ginseng, or Gasiogapi in Korea. A lot of studies reported that $A$. senticosus and A. koreanum contained various bioactive constituents, including triterpenoid saponins, lignans, coumarins, flavones and phenolic compounds [6,7]. The elutheroside $\mathrm{B}$ and $\mathrm{E}$ are major active lignans of $A$. senticosus and $A$. koreanum, which have immunomodulatory, antioxidant and anti-inflammatory activities [6]. In addition, other phenolic compounds contained these herbal plants, such as caffeic acid and chlorogenic acid also showed strong antioxidant ability in vitro and in vivo assays $[8,9]$. Recently, our previous study found that the stem of $A$. senticosus and A. koreanum contained higher amounts of lignans (eleutheroside B and E) than other parts of their plants [10]. The extracts of root, fruit, and stem are commonly used as ingredients in healthcare and medicinal products. Moreover, other parts of plants, such as the leaf, also could be useful in the food and pharmaceutical industries because of the high amount of their constituents.

To evaluate the quality of herbal medicines, reliable analytical methods have to be applied to the quantitative determination of the constituents with known therapeutic activity or to the final products [11]. Phenolic compounds, especially chlorogenic acid and caffeic acid, are major active compounds from A. senticosus and A. koreanum, however, few studies have determined the chlorogenic acid and caffeic acid content of $A$. senticosus $[12,13]$. Therefore, there is need to quantify it by validated methods in these herbal plants. The purpose of this study was to investigate antioxidant activities and correlation between antioxidant activities and total phenolic, flavonoid, and proanthocyanidin contents from different parts of $A$. senticosus and A. koreanum, and to determined chlorogenic acid and caffeic acid contents in the various parts of these plants. In addition, the chlorogenic acid and caffeic acid from the leaf of A. senticosus were validated using high performance liquid chromatography (HPLC).

\section{Results and Discussion}

\subsection{Antioxidant Activity from Various Parts of A. Senticosus and A. Koreanum Extracts}

Reactive oxygen species (ROS), defined as the imbalance between ROS and antioxidants in favor of the oxidants, has been suggested to be the cause of aging and various diseases in humans. Therefore, antioxidant therapy is vital in scavenging free radicals [14]. The antioxidant potential of various extracts of A. senticosus and A. koreanum was determined by the 2,2-diphenyl-1-picrylhydrazyl (DPPH), 
[2,2'-azino-bis (3-ethylbenzothiazoline-6-sulfonic acid)] (ABTS), ferric reducing antioxidant power (FRAP), reducing power and oxygen radial absorbance capacity (ORAC) assays. As shown in Figure 1, the fruit extracts of $A$. koreanum have markedly higher ABTS, FRAP, reducing power and ORAC activities than those of other parts of $A$. senticosus and $A$. koreanum extracts at the concentration of $1 \mathrm{mg} / \mathrm{mL}$. However, DPPH radical scavenging activity of various extracts exhibited that the leaf extracts of $A$. senticosus are much greater than other part extracts. The mechanisms of antioxidant action in radical scavenging assay differed from those in the FRAP, reducing, and ORAC assays [15]. Similar results have reported that the three tropical fruits and wild herb extracts also showed different antioxidant ability with different reaction mechanisms $[15,16]$. Therefore, the evaluation of antioxidant activity is a rather difficult task when a single method is selected. For that reason, the antioxidant activity of samples must be evaluated with a variety of methods that can address the different mechanisms.
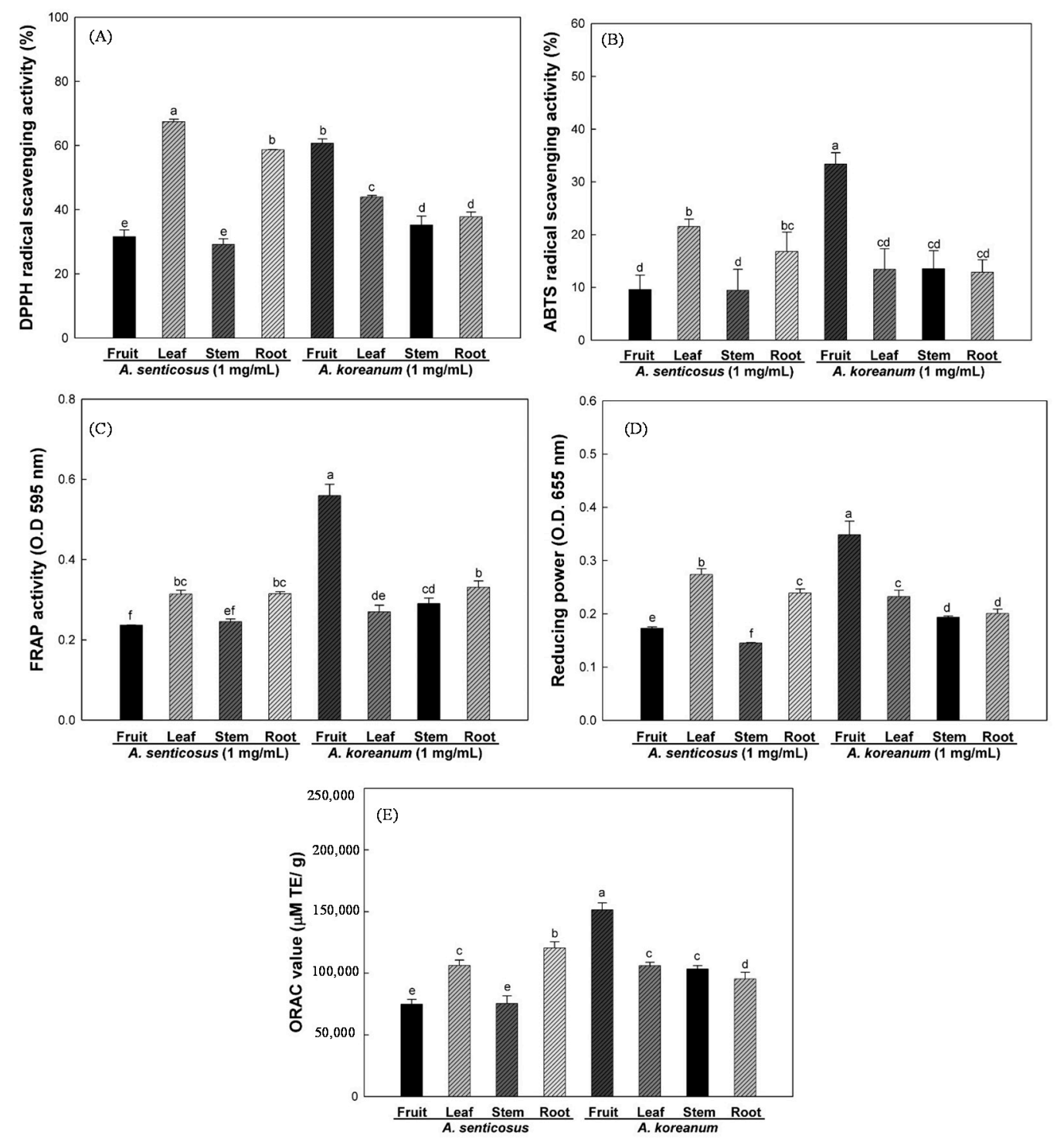

Figure 1. DPPH radical scavenging activity (A); ABTS radical scavenging activity (B); FRAP activity (C); Reducing power (D); and ORAC (E) in the different parts of A. senticosus and $A$. koreanum. Each bar represents the mean $\pm \mathrm{SD}$ of triplicate determinations, $n=3$. Statistical analysis was performed using one-way ANOVA $(p<0.05)$. 


\subsection{Total Phenolic, Flavonoid and Proanthocyanidin Contents}

Plants have been reported to be rich sources of phytochemicals such as phenolics, flavonoids, and proanthocyanidin, and it has been suggested various health benefits like antioxidant activity $[17,18]$. In addition, Cho et al. [19] reported that various antioxidant activities from natural resources were significantly correlated with their major compound contents, such as polyphenol and flavonoid. Therefore, we determined the total phenolic, flavonoid, and proanthocyanidin contents of extracts obtained from various parts of $A$. senticosus and $A$. koreanum. Table 1 shows the total phenolic content of extracts from different parts of $A$. senticosus and A. koreanum. Among the different extracts, the highest phenolic content (73.34 mg GAE/dry weight $\mathrm{g}$ ) was observed from fruit extracts of $A$. koreanum followed by leaf extracts (56.08 mg GAE/dry weight g), root extracts (44.00 mg GAE/dry weight $\mathrm{g}$ ) of $A$. senticosus and leaf extracts (40.61 mg GAE/dry weight $\mathrm{g}$ ) of A. koreanum, however, stem extracts of A. senticosus exhibited the lowest phenolic contents ( $23.50 \mathrm{mg}$ GAE/dry weight $\mathrm{g}$ ). On the other hand, the leaf extracts of A. senticosus (41.23 mg RE/dry weight g) and A. koreanum (38.21 mg RE/dry weight g) showed higher total flavonoid contents than other part extracts of their plants. In all parts of A. senticosus, total phenolic and flavonoid contents followed the same trend; leaf $>$ root $>$ fruit $>$ stem. In another study, the highest amount of total phenolic and flavonoid contents also observed from leaves extracts of A. senticosus [20]. In addition, the total phenolic contents of the leaf extracts from Withania somnifera (L.) also exhibited as remarkably higher than in the other parts of the plant [21]. Proanthocyanidin content in the fruit extracts of $A$. senticosus (10.86 mg CE/dry weight g) and A. koreanum (33.01 mg CE/dry weight g) was higher than other parts (Table 1). Total content of phenolic, flavonoid, and proanthocyanidin influence antioxidant ability [19,22]. Many medicinal plants contain large amounts of antioxidants such as polyphenols [23], and the antioxidant activity of the extracts from various plants might be correlated with their total phenolic compounds [19]. Therefore, the antioxidant activities in different parts of $A$. senticosus and A. koreanum were correlated with total phenolic, flavonoid, and proanthocyanidin contents.

Table 1. Total phenolic, total flavonoid and proanthocyanidin contents in the different parts of $A$. senticosus and A. koreanum.

\begin{tabular}{ccccc}
\hline Sample & & $\begin{array}{c}\text { Total Phenolic Contents } \\
\text { (mg GAE/dry weight g) }\end{array}$ & $\begin{array}{c}\text { Total Flavonoid Contents } \\
\text { (mg RE/dry weight g) }\end{array}$ & $\begin{array}{c}\text { Proanthocyanidin } \\
\text { (mg CE/dry weight g) }\end{array}$ \\
\hline \multirow{6}{*}{ A. senticosus } & Fruit & $25.70 \pm 0.69^{\mathrm{f}}$ & $16.44 \pm 0.99^{\mathrm{g}}$ & $10.86 \pm 0.49^{\mathrm{c}}$ \\
& Leaf & $56.08 \pm 0.47^{\mathrm{b}}$ & $41.23 \pm 1.98^{\mathrm{a}}$ & $8.75 \pm 1.02^{\mathrm{d}}$ \\
& Stem & $23.50 \pm 0.42^{\mathrm{g}}$ & $11.49 \pm 1.49^{\mathrm{h}}$ & $2.88 \pm 0.28^{\mathrm{f}}$ \\
& Root & $44.00 \pm 0.14^{\mathrm{c}}$ & $36.49 \pm 0.37^{\mathrm{b}, \mathrm{c}}$ & $7.61 \pm 0.56^{\mathrm{e}}$ \\
\hline \multirow{6}{*}{ A. koreanum } & Fruit & $73.34 \pm 1.16^{\mathrm{a}}$ & $31.31 \pm 0.37^{\mathrm{d}}$ & $33.01 \pm 1.02^{\mathrm{a}}$ \\
& Leaf & $40.61 \pm 1.03^{\mathrm{d}}$ & $38.21 \pm 0.65^{\mathrm{b}}$ & $6.79 \pm 0.28^{\mathrm{e}}$ \\
& Stem & $35.45 \pm 0.47^{\mathrm{e}}$ & $21.18 \pm 0.99^{\mathrm{f}}$ & $7.44 \pm 0.98^{\mathrm{e}}$ \\
& Root & $34.40 \pm 0.69^{\mathrm{e}}$ & $23.56 \pm 1.63^{\mathrm{e}}$ & $3.86 \pm 0.28^{\mathrm{f}}$ \\
\hline
\end{tabular}

GAE, gallic acid equivalent. RE, rutin equivalent. $\mathrm{CE}$, catechin equivalent. Value are Mean $\pm \mathrm{SD}$ in triplicate $(n=3)$, means in the same column not sharing a common letter are significantly different $(p<0.05)$ by Duncan's multiple test. abcdefg significant differences among various parts of samples. 


\subsection{Relationship between Antioxidant Activity and Antioxidant Compounds}

To correlate the results obtained by the different methods, a regression analysis was performed (correlation coefficient $=\mathrm{R}$ ). In the Table 2, among the various methods used to determine the antioxidant potential, the ABTS radical scavenging activity was significantly correlated with reducing power $(\mathrm{R}=$ 0.966), FRAP $(\mathrm{R}=0.936)$ and ORAC $(\mathrm{R}=0.911)$ assays, however, a low correlation was observed between DPPH and FRAP $(\mathrm{R}=0.580)$. The obtained results also significantly correlated to the total phenolic content with reducing power $(\mathrm{R}=0.992)$, ABTS $(\mathrm{R}=0.981)$ and ORAC $(\mathrm{R}=0.922)$ assays, and proanthocyanidin was highly correlated with FRAP $(\mathrm{R}=0.896)$ and ABTS $(\mathrm{R}=0.877)$ assays. On the other hand, the total flavonoid content was correlated with DPPH $(\mathrm{R}=0.866)$, and ABTS $(\mathrm{R}=0.539)$, FRAP $(\mathrm{R}=0.321)$, reducing power $(\mathrm{R}=0.717)$ and $\mathrm{ORAC}(\mathrm{R}=0.637)$ assays were uncorrelated with total flavonoid contents. Dudonne et al. [24] reported that the total phenolic contents from 30 types of plants significantly correlated with ABTS assay. In general, the antioxidant activity of plant extracts was correlated with their major compounds, such as phenolic compounds, flavonoids, carotenoids, and pigments [25]. These results imply that considerable antioxidant activity except DPPH radical assay from $A$. senticosus and A. koreanum may be due to total phenolic and proanthocyanidin contents rather than total flavonoid contents. Therefore, we determined major active compounds of different parts extracts from $A$. senticosus and A. koreanum using HPLC analysis.

Table 2. Correlation analysis $(\mathrm{R})$ between the antioxidant activity and antioxidant compounds in the different parts of A. senticosus and A. koreanum.

\begin{tabular}{ccccccccc}
\hline Parameter & TPC & TFC & PC & DPPH & ABTS & FRAP & Reducing Power & ORAC \\
\hline TPC & 1 & 0.688 & $0.802 *$ & $0.867 * *$ & $0.981 * *$ & $0.876 * *$ & $0.992 * *$ & $0.922 * *$ \\
TFC & 0.688 & 1 & 0.218 & $0.866 * *$ & 0.539 & 0.321 & $0.717 *$ & 0.637 \\
PC & $0.802 *$ & 0.218 & 1 & 0.480 & $0.877 * *$ & $0.896 * *$ & $0.812 *$ & $0.769 *$ \\
DPPH & $0.867 * *$ & $0.866 * *$ & 0.480 & 1 & $0.792 *$ & 0.580 & $0.861 * *$ & $0.759 *$ \\
ABTS & $0.981 * *$ & 0.539 & $0.877 * *$ & $0.792 *$ & 1 & $0.936 * *$ & $0.966 * *$ & $0.911 * *$ \\
FRAP & $0.876 * *$ & 0.321 & $0.896 * *$ & 0.580 & $0.936 * *$ & 1 & $0.869 * *$ & $0.883 * *$ \\
Reducing power & $0.992 * *$ & $0.717 *$ & $0.812 *$ & $0.861 * *$ & $0.966 * *$ & $0.869 * *$ & 1 & $0.927 * *$ \\
ORAC & $0.922 * *$ & 0.637 & $0.769 *$ & $0.759 *$ & $0.911 * *$ & $0.883 * *$ & $0.927 * *$ & 1 \\
\hline
\end{tabular}

TPC, Total phenolic contents. TFC, Total flavonoid contents. PC, Proanthocyanidin. * The correlation coefficients are significant at the 0.05 level. ** The correlation coefficients are significant at the 0.01 level.

\subsection{The Chlorogenic Acid and Caffeic Acid Contents}

The chlorogenic acid and caffeic acid are major compounds in Acanthopanax species [6]. The contents of chlorogenic acid and caffeic acid in different parts of $A$. senticosus and A. koreanum were analyzed using the developed HPLC method. As a result, chlorogenic acid and caffeic acid were found in extracts from leaf of $A$. senticosus, however, the peak areas corresponding to $p$-coumaric acid and ferulic acid were not found in the sample (Figure 2). Therefore, we determined chlorogenic acid and caffeic acid content in the current study. Table 3 showed chlorogenic acid and caffeic acid contents in different parts of $A$. senticosus and A. koreanum. The highest contents of chlorogenic acid and caffeic acid were observed from leaf extracts of $A$. senticosus. Caffeic acid in fruit and stem extracts of $A$. senticosus and fruit extracts of $A$. koreanum was not detected. The chlorogenic acid contents of leaves extracts from 
A. senticosus $(14.86 \mathrm{mg} / \mathrm{g})$ and A. koreanum $(5.43 \mathrm{~m} / \mathrm{g})$ were significantly greater than leaves extracts of $A$. senticosus from Mudanjiang $(1.1 \mathrm{mg} / \mathrm{g})$ and Wudalianchi $(0.23 \mathrm{mg} / \mathrm{g})$ in Heilongjiang province, China [13]. These results suggest that the chlorogenic acid and caffeic acid contents of Acanthopanax species may be significantly different in their growing environmental factors such as temperature, light, water, and nutrient availability [26].

The obtained results indicate that the strong antioxidant activities and highest amount of chlorogenic acid and caffeic acid contents from leaf of $A$. senticosus could be a good source for natural antioxidant products. Therefore, the major phenolic compounds were validated by HPLC method from leaf extracts of $A$. senticosus.
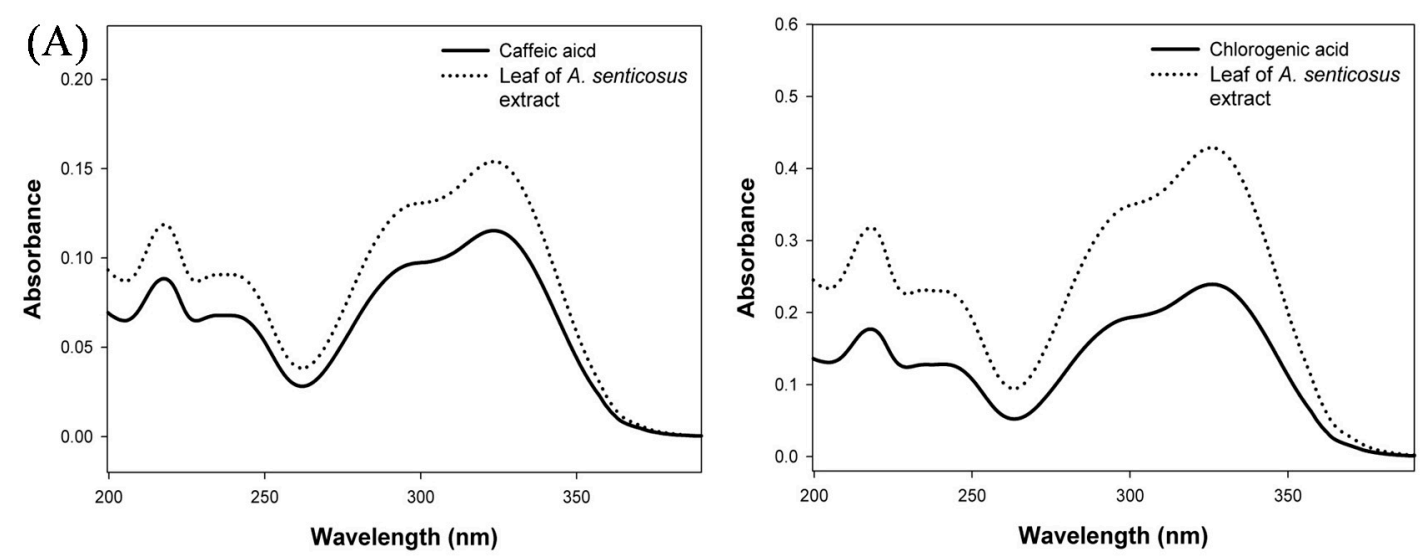

(B)
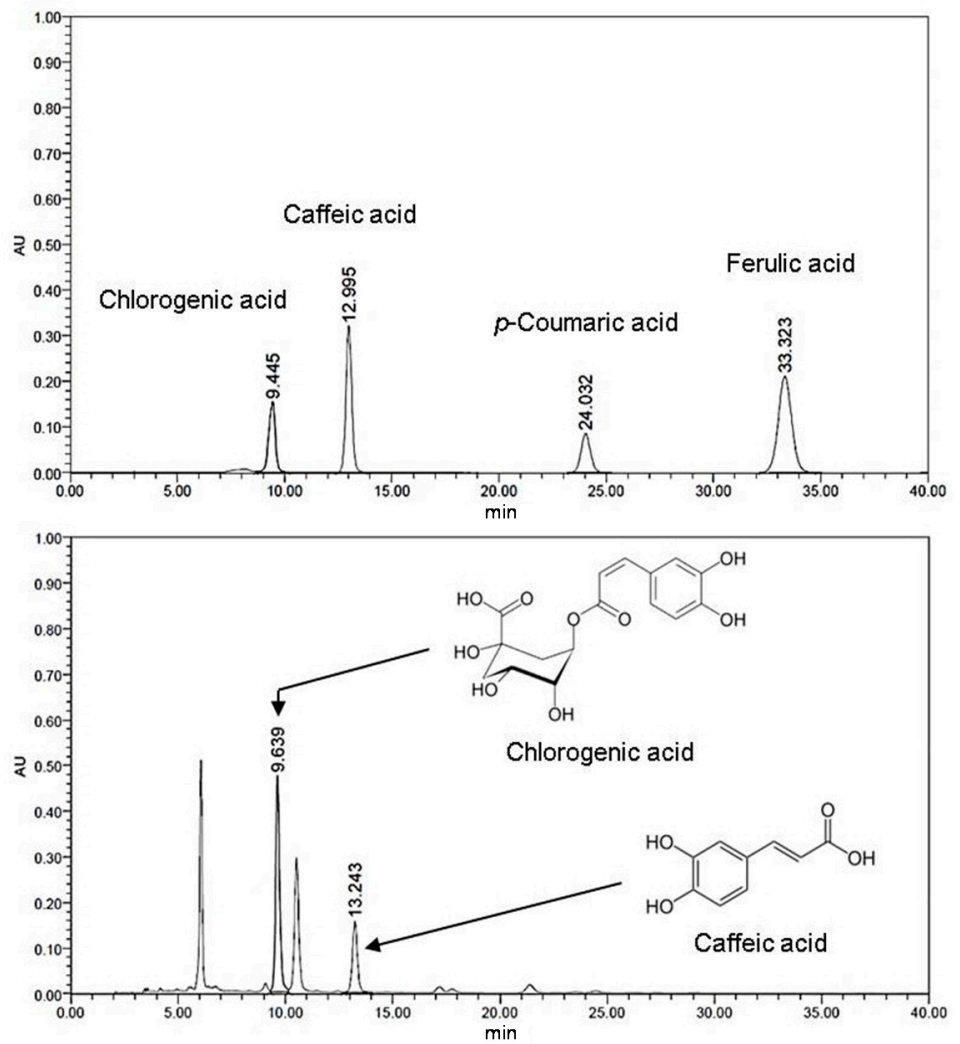

Figure 2. PDA spectrums of caffeic acid and chlorogenic acid (A); and HPLC chromatograms of phenolic compounds (chlorogenic acid, caffeic acid, $p$-coumaric acid, and ferulic acid) (B) of leaf extract from $A$. senticosus. 
Table 3. Chlorogenic acid and caffeic acid contents in the different parts of A. senticosus and A. koreanum.

\begin{tabular}{|c|c|c|c|}
\hline Sampl & & $\begin{array}{l}\text { Chlorogenic Acid } \\
\text { (mg/dry weight g) }\end{array}$ & $\begin{array}{c}\text { Caffeic Acid } \\
\text { (mg/dry weight g) }\end{array}$ \\
\hline \multirow{4}{*}{ A. senticosus } & Fruit & $0.57 \pm 0.01^{\mathrm{f}}$ & Not detected \\
\hline & Leaf & $14.86 \pm 0.12^{\mathrm{a}}$ & $3.09 \pm 0.02^{\mathrm{a}}$ \\
\hline & Stem & $6.02 \pm 0.12^{b}$ & Not detected \\
\hline & Root & $5.01 \pm 0.20^{\mathrm{d}}$ & $0.10 \pm 0.03^{\mathrm{d}}$ \\
\hline \multirow{4}{*}{ A. koreanum } & Fruit & $1.92 \pm 0.03^{\mathrm{e}}$ & Not detected \\
\hline & Leaf & $5.43 \pm 0.16^{c}$ & $1.61 \pm 0.07^{\mathrm{b}}$ \\
\hline & Stem & $5.96 \pm 0.07^{b}$ & $1.22 \pm 0.04^{\mathrm{c}}$ \\
\hline & Root & $6.14 \pm 0.14^{b}$ & $0.11 \pm 0.01^{\mathrm{d}}$ \\
\hline
\end{tabular}

Value are Mean \pm SD in triplicate $(n=3)$, means in the same column not sharing a common letter are significantly different $(p<0.05)$ by Duncan's multiple test. ${ }^{\text {abcdef }}$ significant differences among various parts of samples.

\subsection{Method Validation}

\subsubsection{Specificity}

As recommended by the Korea food and drug administration (KFDA) guidelines [27], a chromatographic method is considered specific (selective) when each peak within the chromatogram corresponds to only one substance. Specificity is the ability to distinguish between the analyte and other substances that may be present in a sample. In general, this analysis is performed by a photo diode array (PDA) detector that provides the chromatogram and ultraviolet (UV) spectral information. As shown in Figure 2, the peak of $A$. senticosus leaf extracts was verified by comparing the retention time in the HPLC chromatogram and the UV spectrum (200-400 nm) of chlorogenic acid and caffeic acid. However, the peak areas corresponding to $p$-coumaric acid and ferulic acid were not found in the leaf extracts from A. senticosus.

\subsubsection{Evaluation of Accuracy and Precision, Limit of Detection, and Limit of Quantification}

The precision of the method was assessed by intra-day and inter-day variations. The method showed good precision, with intra-day and inter-day variations of $0.87 \%-1.39 \%$ (RSD \%) and $1.47 \%-1.70 \%$, respectively (Table 4). To evaluate the accuracy of the method, as shown in Table 5, a recovery study was carried out by spiking $A$. senticosus leaf extracts at three concentrations $(25 \%, 50 \%$ and $100 \%$ of A. senticosus leaf extracts) of chlorogenic acid and caffeic acid. The mean recovery for chlorogenic acid ranged from $105.01 \%-110.74 \%$, while the percentage of relative standard deviation (RSD) for recovery was $0.92 \%-3.10 \%$. The mean recovery for caffeic acid ranged from $91.29 \%-96.41 \%$, while the percentage of RSD for recovery was $0.52 \%-1.91 \%$. Thus, the developed method was considered to be reproducible and accurate (Table 5). Based on these results, a simple and reliable RP-HPLC method with photodiode assay detector for the quantification of chlorogenic acid and caffeic acid has been developed and validated for its specificity, linearity, accuracy, precision, limits of detection (LOD), and limits of quantification (LOQ). 
The calibration curve showed excellent linearity (correlation coefficients $(\mathrm{R})$ : chlorogenic acid and caffeic acid $=0.9997$ and 0.9991 , respectively). The LOD and LOQ values of the chlorogenic acid were $0.10 \mu \mathrm{g} / \mathrm{mL}$ and $0.30 \mu \mathrm{g} / \mathrm{mL}$, respectively, and the LOD and LOQ values of the caffeic acid were $0.03 \mu \mathrm{g} / \mathrm{mL}$ and $0.06 \mu \mathrm{g} / \mathrm{mL}$, respectively (Table 5).

Table 4. Precision studies of chlorogenic acid and caffeic acid analysis for the determination in the leaf extract of $A$. senticosus.

\begin{tabular}{ccccc}
\hline \multirow{2}{*}{ Parameters } & \multicolumn{2}{c}{ Chlorogenic Acid (mg/dry weight g) } & \multicolumn{2}{c}{ Caffeic Acid (mg/dry weight g) } \\
\cline { 2 - 5 } & Mean \pm SD & RSD (\%) & Mean \pm SD & RSD (\%) \\
\hline Inter-day & $15.15 \pm 0.13$ & 0.87 & $3.32 \pm 0.05$ & 1.39 \\
Intra-day & $15.56 \pm 0.26$ & 1.70 & $3.43 \pm 0.05$ & 1.47 \\
\hline
\end{tabular}

Results are expressed as the mean \pm standard deviation (SD) from at least three independent experiments performed in triplicate. RSD, Relative standard deviation. Inter-day, One time analysis of chlorogenic acid and caffeic acid per day for 3 days. Intra-day, Three times analysis per day.

Table 5. The recovery, LOD and LOQ values of chlorogenic acid and caffeic acid in the leaf extract from A. senticosus.

\begin{tabular}{cccccc}
\hline \multirow{2}{*}{ Added (mg/dry weight g) } & \multicolumn{2}{c}{ Recovery (\%) } & \multicolumn{2}{c}{ Values of LOD and LOQ $(\boldsymbol{\mu g} / \mathbf{m L})$} \\
\cline { 3 - 6 } & & Mean \pm SD & RSD (\%) & LOD & LOQ \\
\hline \multirow{3}{*}{ Chlorogenic acid } & $15.15(100 \%)$ & $110.74 \pm 2.84$ & 2.56 & - & - \\
& $7.58(50 \%)$ & $105.01 \pm 0.97$ & 0.92 & - & - \\
& $3.79(25 \%)$ & $106.00 \pm 3.29$ & 3.10 & 0.1 & 0.3 \\
\hline \multirow{3}{*}{ Caffeic acid } & $3.32(100 \%)$ & $96.41 \pm 1.84$ & 1.91 & - & - \\
& $1.66(50 \%)$ & $92.49 \pm 0.75$ & 0.81 & - & 0.06 \\
\hline
\end{tabular}

Results are expressed as the mean \pm standard deviation (SD) from at least three independent experiments performed in triplicate. RSD, Relative standard deviation.

\section{Experimental Section}

\subsection{Sample Preparation}

A. senticosus and A. koreanum were supplied by farms located in Gangwon and Jeju provinces, Korea, respectively. Dried fruit, leaf, stem, and root of A. senticosus and A. koreanum were ground in a mill and passed through a 35-mesh sieve. Approximately $3 \mathrm{~g}$ of dry powdered plant material was extracted with $100 \mathrm{~mL}$ water by reflux for $3 \mathrm{~h}$ at $100^{\circ} \mathrm{C}$. After filtration using filter paper (Whatman No. 3, Maidstone, Kent, UK), the extract was concentrated by evaporation under a vacuum at $60{ }^{\circ} \mathrm{C}$ in a rotary vacuum evaporator (Tokyo Rikakikai Co., Ltd, Tokyo, Japan) and then freeze-dried (Ilshinbiobase Co., Ltd, Gyeonggi, Korea).

\subsection{Chemicals}

The reagents sodium carbonate, aluminum nitrate, potassium ferricyanide, sodium hydroxide, Folin-Ciocalteu's phenol reagent, vanillin, potassium acetate, Trolox, potassium persulfate, sodium 
phosphate dibasic, trichloroacetic acid, ferric chloride, phosphoric acid, gallic acid, rutin, catechin, chlorogenic acid, caffeic acid, $p$-coumaric acid, ferulic acid, DPPH (2,2-diphenyl-1-picrylhydrazyl), ABTS [2,2'-azino-bis (3-ethylbenzothiazoline-6-sulfonic acid)], TPTZ (2,4,6-tripyridyl-s-triazine) and AAPH (2,2'-azobis (2-methylpropionamidine) dihydrochloride) were purchased from Sigma Chemical Co. (St Louis, MO, USA). Potassium phosphate monobasic and fluorescein (sodium salt) were purchased from Junsei Chemical Co. (Tokyo, Japan), and HPLC-grade water and acetonitrile were obtained from J. T. Baker (Phillipsburg, NJ, USA).

\subsection{Antioxidant Activity}

\subsubsection{DPPH Radical Scavenging Activity}

Antioxidant activity determination of the different extracts was performed by the DPPH radical scavenging method [28]. DPPH radicals have an absorption maximum of $517 \mathrm{~nm}$, which disappears with reduction by an antioxidant compound. Water solution $(1 \mathrm{~mL})$ containing $1 \mathrm{mg}$ of the freeze-dried extract $(0.2 \mathrm{~mL})$ was added to a $0.4 \mathrm{mM} \mathrm{DPPH}$ ethanolic solution $(0.8 \mathrm{~mL})$. The solution was mixed and allowed to react at room temperature in the dark for $10 \mathrm{~min}$. The absorbance at $517 \mathrm{~nm}$ was measured using a microplate reader. The blank was prepared in the same manner, except that distilled water was used instead of the sample. The radical scavenging activity was calculated as a percentage using the following equation:

$$
\text { DPPH radical scavenging activity }(\%)=\left[1-\left(\mathrm{A}_{\text {sample }} / \mathrm{A}_{\text {blank }}\right)\right] \times 100
$$

\subsubsection{ABTS Radical Scavenging Activity}

ABTS radical scavenging activity of the different extracts was measured according to the modified method of Re et al. [29]. ABTS stock solution was dissolved in water to a $7.4 \mathrm{mM}$ concentration. The ABTS radical cation $\left(\mathrm{ABTS}^{+}\right)$was produced by reacting ABTS stock solution with $2.45 \mathrm{mM}$ potassium persulfate and allowing the mixture to stand for $14 \mathrm{~h}$ at room temperature in the dark. The ABTS ${ }^{+}$ solution was diluted with ethanol to obtain an absorbance of $0.70 \pm 0.02$ at $750 \mathrm{~nm}$. After adding $1.0 \mathrm{~mL}$ of diluted $\mathrm{ABTS}^{+}$solution $(\mathrm{A} 750 \mathrm{~nm}=0.70 \pm 0.02)$ to $0.01 \mathrm{~mL}$ of sample, the mixture was left at room temperature for $30 \mathrm{~min}$ in the dark. The absorbance at $750 \mathrm{~nm}$ was measured using a microplate reader. The blank was prepared in the same manner, except distilled water was used instead of the sample. The radical scavenging activity was calculated as a percentage using the following equation:

$$
\text { ABTS radical scavenging activity }(\%)=\left[1-\left(\mathrm{A}_{\text {sample }} / \mathrm{A}_{\text {blank }}\right)\right] \times 100
$$

\subsubsection{Ferric Reducing Antioxidant Power (FRAP)}

The FRAP assay was performed using a modified version of the FRAP assay [30]. The working FRAP reagent was prepared daily by $300 \mathrm{mM}$ acetate buffer ( $\mathrm{pH}$ 3.6), a $10 \mathrm{mM}$ TPTZ solution in $40 \mathrm{mM} \mathrm{HCl}$ and $20 \mathrm{mM} \mathrm{FeCl}_{3} \cdot 6 \mathrm{H}_{2} \mathrm{O}$ solution in proportions of 10:1:1 (v/v), respectively. The FRAP reagent was prepared fresh daily and was warmed to $37^{\circ} \mathrm{C}$ in a water bath prior to use. Then, $0.05 \mathrm{~mL}$ of sample was mixed with distilled water $(0.15 \mathrm{~mL})$ and the FRAP reagent $(1.5 \mathrm{~mL})$. The reaction 
mixture was incubated at $37{ }^{\circ} \mathrm{C}$ in a water bath for $4 \mathrm{~min}$, and the absorbance of the colored products was measured at $595 \mathrm{~nm}$.

\subsubsection{Reducing Power}

The reducing power of the sample was determined according to the modified method of reducing power assay [31]. This assay is based on reduction; the yellow color of the solution changes to various shades of green and blue depending on the reducing power of sample. The sample $(0.1 \mathrm{~mL})$ was added to $0.2 \mathrm{M}$ sodium phosphate buffer $(0.5 \mathrm{~mL})$ and $1 \%$ potassium ferricyanide $(0.5 \mathrm{~mL})$, and this mixture was incubated at $50{ }^{\circ} \mathrm{C}$ for $20 \mathrm{~min}$. Following incubation, $10 \%$ trichloroacetic acid solution $(0.5 \mathrm{~mL})$ was added to the reaction mixture, and it was centrifuged at $12,000 \mathrm{rpm}$ for $10 \mathrm{~min}$. The supernatant was mixed with distilled water $(0.5 \mathrm{~mL})$ and a $0.1 \%$ iron (III) chloride solution $(0.1 \mathrm{~mL})$, and the absorbance at $700 \mathrm{~nm}$ of the resulting solution was measured.

\subsubsection{Oxygen Radical Absorbance Capacity (ORAC)}

The ORAC assay is based on the scavenging of peroxyl radicals generated by AAPH, which prevent the degradation of the fluorescein probe. The ORAC assay was performed using a modified method of Ou et al. [32]. The sample was diluted with a $75 \mathrm{mM}$ potassium sodium phosphate buffer ( $\mathrm{pH} 7.4)$, and $0.025 \mathrm{~mL}$ of either the diluted Trolox $(0-10 \mu \mathrm{M})$ or the diluted sample, together with $150 \mu \mathrm{L}$ of $40 \mathrm{nM}$ fluorescein, was added into black-walled 96-well plate. Finally, $25 \mu \mathrm{L}$ of $18 \mathrm{mM}$ AAPH was pre-incubated at $37^{\circ} \mathrm{C}$ for $15 \mathrm{~min}$ and transferred to each well, and the plate was immediately carried to the fluorescence microplate reader (Spectramax GEMINI EM, Molecular Devices, Sunnyvale, CA, USA) to measure the fluorescence. The analyzer was set to an excitation wavelength of $485 \mathrm{~nm}$ and an emission wavelength of $530 \mathrm{~nm}$, and readings were recorded every $3 \mathrm{~min}$ for $90 \mathrm{~min}$ at $37^{\circ} \mathrm{C}$. The ORAC value results were calculated using a Trolox calibration curve and the area under the fluorescence decay curve. The ORAC value was expressed as Trolox equivalents in micromoles per milliliter.

Area under the curve $(\mathrm{AUC})=1+\mathrm{f} 1 / \mathrm{f} 0+\mathrm{f} 2 / \mathrm{f} 0+\mathrm{f} 3 / \mathrm{f} 0+\mathrm{f} 4 / \mathrm{f} 0+\ldots \mathrm{f} 31 / \mathrm{f} 0$

\subsubsection{Total Phenolic Content (TPC)}

The total phenolic content was determined using Folin-Ciocalteu assay [33]. Water solution (1 mL) containing $1 \mathrm{mg}$ of the freeze-dried extract or standard was mixed with $1 \mathrm{~mL}$ of $2 \%$ sodium carbonate solution and $1 \mathrm{~mL}$ of 10\% Folin-Ciocalteu's phenol reagent. After $90 \mathrm{~min}$, the absorbance was measured at $750 \mathrm{~nm}$ using a microplate reader (Molecular Devices, Sunnyvale, CA, USA). The measurement was compared to calibration curve of gallic acid [total phenolic concentration $=($ absorbance -0.0136$) / 15.177$ $\times$ extraction yield; $\left.\mathrm{R}^{2}=0.996 ; p<0.001\right]$, and the results were expressed as milligrams of gallic acid equivalents (GAE) per gram of sample $[\mathrm{mg} \mathrm{GAE} / \mathrm{g}]$.

\subsubsection{Total Flavonoid Content (TFC)}

The total flavonoid content was determined using a method described by Zhishen et al. [34] with modifications. Water solution $(0.5 \mathrm{~mL})$ containing $0.5 \mathrm{mg}$ of the freeze-dried extract was mixed with $1.5 \mathrm{~mL}$ of ethanol, $0.1 \mathrm{~mL}$ of $10 \%$ aluminum nitrite solution, $0.1 \mathrm{~mL}$ of $1 \mathrm{M}$ potassium acetate solution 
and $2.8 \mathrm{~mL}$ distilled water. The mixture was stirred and allowed to react for $30 \mathrm{~min}$. The absorbance was then measured at $415 \mathrm{~nm}$ using a microplate reader. The measurement was compared to a calibration curve of rutin [flavonoid concentration $=($ absorbance +0.0001$) / 1.5467 \times$ extraction yield; $\mathrm{R}^{2}=0.999$; $p<0.001$ ], and the results were expressed as milligrams of rutin equivalents (RE) per gram of sample $[\mathrm{mg} \mathrm{RE} / \mathrm{g}]$.

\subsubsection{Proanthocyanidin Content (PC)}

The proanthocyanidin content was determined using vanillin-hydrochloric acid (V-HCl) method [35]. Methanol solution $(0.5 \mathrm{~mL})$ containing $0.5 \mathrm{mg}$ of freeze-dried extract was placed in a brown tube, and $3 \mathrm{~mL}$ of $4 \%$ vanillin solution was added. After stirring vigorously, $1.5 \mathrm{~mL}$ of concentrated hydrochloric acid was added to the mixture and was allowed to react for $15 \mathrm{~min}$. The absorbance was then measured at $490 \mathrm{~nm}$ using a microplate reader. The measurement was compared to a calibration curve of catechin [proanthocyanidin concentration $=($ absorbance -0.0011$) / 2.0467 \times$ extraction yield; $\mathrm{R}^{2}=0.999$; $p<0.001]$, and the results were expressed as milligrams of catechin equivalents (CE) per gram of sample $[\mathrm{mg} \mathrm{CE} / \mathrm{g}]$.

\subsection{HPLC Analysis of Phenolic Compounds}

Analysis of the phenolic compounds was carried out with an HPLC system (Waters 2695 Separation Module, Waters Co., Milford, MA, USA) controlled by Empower software. Polyphenol separation was determined using a modified method of Li et al. [12].

For the simultaneous analysis of chlorogenic acid, caffeic acid, $p$-coumaric acid and ferulic acid were identified by comparing the retention times and UV spectra with those obtained with standard mixture. The phenolic compounds were quantified by the external standard method with calibration curves (correlation coefficients $>0.999$ for chlorogenic acid and caffeic acid).

\subsection{Validation Procedure}

The HPLC method was validated in terms of linearity, specificity, limit of detection (LOD), limit of quantification (LOQ), precision, and accuracy according to the guidelines of KFDA (Korea Food \& Drug Administration) [27].

\subsubsection{Specificity}

The specificity of the method was assessed by comparing the chromatograms (HPLC) and PDA spectra (UV) obtained from the standard phenolic compounds and the leaf extract from A. senticosus.

\subsubsection{Linearity}

For the linearity study, calibration curves were carried out with five concentration levels for each target. Each concentration was analyzed three times. The calibration curve was quantified as $\mathrm{y}=\mathrm{Ax}+\mathrm{B}$, where " $\mathrm{A}$ " is the slope of the calibration curve, " $\mathrm{B}$ " is the intercept of the calibration curve, " $\mathrm{x}$ " is the concentration of the phenolic compound in standard solution, and " $y$ " is the peak area. The correlation (R) value indicated linearity. 


\subsubsection{Precision and Accuracy}

Precision was evaluated by measuring analysis repeatability in the intra- and inter-day tests. The samples were injected three times, and the results were expressed as the Relative Standard Deviation of measurements (RSD \%).

Accuracy was validated through a spike recovery test. To perform this test, sample solutions were prepared by adding phenolic compound at three different concentration levels $(25 \%, 50 \%, 100 \%$ of phenolic compound from $A$. senticosus leaf extract). The accuracy was expressed as the percentage of the amount recovered in the spiked compared to the known concentration.

\subsubsection{Limit of Detection (LOD) and Limit of Quantification (LOQ)}

The LOD is defined as the lowest concentration of an analyte that an analytical process can reliably differentiate from background levels. Additionally, the lowest concentration that can be quantified with acceptable accuracy and precision is defined as LOQ. The LOD and LOQ of the HPLC method were estimated from the signal-to-noise ratio $(\mathrm{S} / \mathrm{N})$. The LOD and LOQ for each analyte were estimated as the concentration levels at which the $\mathrm{S} / \mathrm{N}$ reached 3 and 10 , respectively.

\subsection{Statistical Analysis}

All data are presented as means $\pm \mathrm{SD}$. The data were analyzed using the one-way ANOVA procedure of SAS 9.3 (SAS Institute Inc., Cary, NC, USA). Differences were analyzed using Duncan's multiple range tests at a $p$-value $<0.05$ level. Correlations were calculated using Pearson's correlation coefficient (R) by the IBM SPSS Statistics software package (IBM SPSS Statistics 21, IMB, NY, USA).

\section{Conclusions}

In this study, the antioxidant activities of different parts extracts from $A$. senticosus and $A$. koreanum were measured, and total phenolic, flavonoid, and proanthocyanidin contents are determined. In addition, the major phenolic compounds (chlorogenic acid and caffeic acid) were validated using HPLC method. The in vitro antioxidant activities observed that fruit extracts from $A$. senticosus and $A$. koreanum showed highest ABTS, FRAP, reducing power and ORAC capacity, however, leaf extract of A. senticosus exhibited strongest DPPH radical scavenging activity. The antioxidant activities from various extracts correlated with total phenolic and proanthocyanidin contents rather than total flavonoid contents. The major phenolic compounds from $A$. senticosus and $A$. koreanum were identified as chlorogenic acid and caffeic acid by HPLC. Moreover, the leaf of $A$. senticosus higher contents of chlorogenic acid and caffeic acid than other part of their plants. Therefore, the leaf of A. senticosus is an excellent natural source for antioxidants and can be utilized to develop functional foods as well as serve health-promoting pharmaceutical agents, and the developed validation method was useful for the quality control of Acanthopanax species. 


\section{Acknowledgement}

This research was supported by a 2012 research grant from small and medium business administration (C1008942-01-01).

\section{Author Contributions}

Y.-H. Kim and M.L. Cho prepared the drafts of the manuscript and data collection. D.-B. Kim, G.-H. Shin, J.-H. Lee and J.S. Lee took part in data collection. S.-O. Park and S.-J. Lee involved in data collection, analyzed on statistical results, and H.M. Shin reviewed and advised the manuscript. O.-H. Lee was responsible for designing the experiment, obtaining the funds for conducting the study, supervising experimental design. All authors read and contributed to the final manuscript.

\section{Conflicts of Interest}

The authors declare no conflict of interest.

\section{References}

1. Chen, C.Y.O.; Ribaya-Mercado, J.D.; McKay, D.L.; Croom, E.; Blumberg, J.B. Differential antioxidant and quinone reductase inducing activity of American, Asian, and Siberian ginseng. Food Chem. 2010, 119, 445-451.

2. Yu, C.Y.; Kim, S.H.; Lim, J.D.; Kim, M.J.; Chung, I.M. Intraspecific relationship analysis by DNA markers and in vitro cytotoxic and antioxidant activity in Eleutherococcus senticosus. Toxicol. in Vitro 2003, 17, 229-236.

3. Lin, Q.Y.; Jin, L.J.; Cao, Z.H.; Li, H.Q.; Xu, Y.P. Protective effect of Acanthopanax senticosus extract against endotoxic shock in mice. J. Ethnopharmacol. 2008, 118, 495-502.

4. Ahn, J.; Um, M.Y.; Lee, H.; Jung, C.H.; Heo, S.H.; Ha, T.Y. Eleutheroside E, an active component of Eleutherococcus senticosus, ameliorates insulin resistance in type 2 diabetic $\mathrm{db} / \mathrm{db}$ mice. Evid. Based Complement. Altern. Med. 2013, 2013, 934183.

5. Rates, S.M.K. Plants as source of drugs. Toxicon 2001, 39, 603-613.

6. Huang, L.; Zhao, H.; Huang, B.; Zheng, C.; Peng, W.; Qin, L. Acanthopanax senticosus: Review of botany, chemistry and pharmacology. Pharmazie 2011, 66, 83-97.

7. Kang, J.S.; Linh, P.T.; Cai, X.F.; Kim, H.S.; Lee, J.J.; Kim, Y.H. Quantitative determination of eleutheroside B and E from Acanthopanax species by high performance liquid chromatography. Arch. Pharm. Res. 2001, 24, 407-411.

8. Olthof, M.R.; Hollman, P.C.H.; Katan, M.B. Chlorogenic acid and caffeic acid are absorbed in humans. J. Nutr. 2001, 131, 66-71.

9. Sato, Y.; Itagaki, S.; Kurokawa, T.; Ogura, J.; Kobayashi, M.; Hirano, T.; Sugawara, M.; Iseki, K. In vitro and in vivo antioxidant properties of chlorogenic acid and caffeic acid. Int. J. Pharm. 2011, 403, 136-138.

10. Kim, Y.H.; Bae, D.B.; Lee, J.S.; Park, S.O.; Lee, S.J.; Cho, O.H.; Lee, O.H. Determination of eleutherosides and $\beta$-glucan content from different parts and cultivating areas of $A$. senticosus and A. koreanum. J. Korean Soc. Food Sci. Nutr. 2013, 42, 2082-2087. 
11. Ong, E.S. Extraction methods and chemical standardization of botanicals and herbal preparations. J. Chromatogr. B Anal. Technol. Biomed. Life Sci. 2004, 812, 23-33.

12. Li, Q.; Jia, Y.; Xu, L.; Wang, X.; Shen, Z.; Liu, Y.; Bi, K. Simultaneous determination of protocatechuic acid, syringin, chlorogenic acid, caffeic acid, liriodendrin and isofraxidin in Acanthopanax senticosus Harms by HPLC-DAD. Biol. Pharm. Bull. 2006. 29, 532-534.

13. Zhou, X.; Zheng, C.; Huang, J.; You, T. Identification of herb Acanthopanax senticosus (Rupr. Et Maxim.) harms by capillary electrophoresis with electrochemical detection. Anal. Sci. 2007, 23, 705-711.

14. Finkel, T.; Holbrook, N.J. Oxidants, oxidative stress and the biology of ageing. Nature 2000, 408, 239-247.

15. Lee, Y.J.; Kim, D.B.; Lee, J.S.; Cho, J.H.; Kim, B.K.; Choi, H.S.; Lee, B.Y.; Lee, O.H. Antioxidant activity and anti-adipogenic effects of wild herbs mainly cultivated in Korea. Molecules 2013, 18, 12937-12950.

16. Moo-Huchin, V.M.; Moo-Huchin, M.I.; Estrada-Leon, R.J.; Cuevas-Glory, L.; Estrada-Mota, I.A.; Ortiz-Vazquez, E.; Betancur-Ancona, D.; Sauri-Duch, E. Antioxidant compounds, antioxidant activity and phenolic content in peel from three tropical fruits from Yucatan, Mexico. Food Chem. 2015, 166, 17-22.

17. Hanasaki, Y.; Ogawa, S.; Fukui, S. The correlation between active oxygens scavenging and antioxidative effects of flavonoids. Free Radic. Biol. Med. 1994, 16, 845-850.

18. Rice-Evans, C.A.; Miller, N.J.; Paganga, G. Structure-antioxidant activity relationships of flavonoids and phenolic acids. Free Radic. Biol. Med. 1996, 20, 933-956.

19. Cho, M.L.; Lee, H.S.; Kang, I.J.; Won, M.H.; You, S.G. Antioxidant properties of extract and fractions from Enteromorpha prolifera, a type of green seaweed. Food Chem. 2011, 127, 999-1006.

20. Heo, S.J.; Ahn, H.Y.; Kang, M.J.; Lee, J.H.; Cha, J.Y.; Cho, Y.S. Antioxidative activity and chemical characteristics of leaves, roots, stems and fruits extracts from Acanthopanax senticosus. J. Life Sci. 2011, 21, 1052-1059.

21. Fernando, I.; Abeysinghe, D.; Dharmadasa, R. Determination of phenolic contents and antioxidant capacity of different parts of Withania somnifera (L.) Dunal. from three different growth stages. Ind. Crops Prod. 2013, 50, 537-539.

22. Lachman, J. Major factors influencing antioxidant contents and antioxidant activity in grapes and wines. Int. J. Wine Res. 2009, 1, 101-121.

23. Adedapo, A.A.; Jimoh, F.O.; Afolayan, A.J.; Masika, P.J. Antioxidant properties of the methanol extracts of the leaves and stems of Celtis africana. Rec. Nat. Prod. 2009, 3, 23-31.

24. Dudonne, S.; Vitrac, X.; Coutiere, P.; Woillez, M.; Merillon, J.M. Comparative study of antioxidant properties and total phenolic content of 30 plant extracts of industrial interest using DPPH, ABTS, FRAP, SOD, and ORAC assays. J. Agric. Food Chem. 2009, 57, 1768-1774.

25. Skerget, M.; Kotnik, P.; Hadolin, M.; Hras, A.R.; Simonic, M.; Knez, Z. Phenols, proanthocyanidins, flavones and flavonols in some plant materials and their antioxidant activities. Food Chem. 2005, 89, 191-198.

26. Dumas, Y.; Dadomo, M.; Lucca, G.D.; Grolier, P. Effects of environmental factors and agricultural techniques on antioxidant content of tomatoes. J. Sci. Food Agric. 2003, 83, 369-382. 
27. KFDA (Korea Food and Drug Administration). Analytical Method Guideline about Validation of Drugs and etc.; Korea Food \& Drug Administration: Osong, Korea, 2012; pp. 1-26.

28. Gadow, A.; Joubert, E.; Hansmann, C.F. Comparison of the antioxidant activity of aspalathin with that of other plant phenols of rooibos tea (Aspalathus linearis), $\alpha$-tocopherol, BHT, and BHA. J. Agric. Food Chem. 1997, 45, 632-638.

29. Re, R.; Pellegrini, N.; Proteggente, A.; Pannala, A.; Yang, M.; Rice-Evans, C. Antioxidant activity applying an improved ABTS radical cation decolorization assay. Free Radic. Biol. Med. 1999, 26, 1231-1237.

30. Benzie, I.F.; Strain, J. The ferric reducing ability of plasma (FRAP) as a measure of "antioxidant power": The FRAP assay. Anal. Biochem. 1996, 239, 70-76.

31. Ferreira, I.C.; Baptista, P.; Vilas-Boas, M.; Barros, L. Free-radical scavenging capacity and reducing power of wild edible mushrooms from northeast Portugal: Individual cap and stipe activity. Food Chem. 2007, 100, 1511-1516.

32. Ou, B.; Hampsch-Woodill, M.; Prior, R.L. Development and validation of an improved oxygen radical absorbance capacity assay using fluorescein as the fluorescent probe. J. Agric. Food Chem. 2001, 49, 4619-4626.

33. Tezcan, F.; Gultekin-Ozguven, M.; Diken, T.; Ozcelik, B.; Erim, F.B. Antioxidant activity and total phenolic, organic acid and sugar content in commercial pomegranate juices. Food Chem. 2009, 115, 873-877.

34. Zhishen, J.; Mengcheng, T.; Jianming, W. The determination of flavonoid contents in mulberry and their scavenging effects on superoxide radicals. Food Chem. 1999, 64, 555-559.

35. Mitsunaga, T.; Doi, T.; Kondo, Y.; Abe, I. Color development of proanthocyanidins in vanillin-hydrochloric acid reaction. J. Wood Sci. 1998, 44, 125-130.

Sample Availability: Samples of different parts extracts from Acanthopanax senticosus and A. senticosus are available from authors.

(C) 2015 by the authors; licensee MDPI, Basel, Switzerland. This article is an open-access article distributed under the terms and conditions of the Creative Commons Attribution license (http://creativecommons.org/licenses/by/4.0/). 